\title{
Monte Carlo study of the domain growth in nonstoichiometric two-dimensional binary alloys
}

\author{
Marcel Porta and Teresa Castán \\ Departament d'Estructura i Constituents de la Matèria, Facultat de Física, Universitat de Barcelona, \\ Diagonal 647, 08028 Barcelona, Catalonia, Spain
}

(Received 8 November 1995)

\begin{abstract}
We use a nearest-neighbor antiferromagnetic Ising model with spin-exchange dynamics to study by Monte Carlo simulations the dynamics of ordering in low-temperature quenched nonstoichiometric $A_{x} B_{1-x}$ binary alloys. By implementing the conserved spin-exchange dynamics into the Monte Carlo method the system evolves so that the density is preserved while the order parameter is not. The simulations have been carried out on a two-dimensional square lattice and the stoichiometric value of the composition $x$ is $x_{0}=0.50$. By using different values of $x$ ranging from $0.60 \leqslant x \leqslant x_{0}=0.50$, we study the influence of the off-stoichiometry on the dynamics of ordering. Regarding the behavior of the excess particles all along the ordering process, we obtain two different regimes. (i) At early to intermediate times the density of excess particles at the interfaces rapidly increases, reaching a saturated value. This density of saturation depends on both composition and temperature. As a consequence of this, since the disorder tends to be localized at the interfaces, the local order inside the growing domains is higher than the equilibrium value. (ii) Once saturation is reached, the system evolves so that the density of excess particles at the interfaces remains constant. During this second regime the excess particles are expelled back to the bulk as the total interface length decreases. We use two different measures for the growth: the total interface length and the structure factor. We obtain that during the second regime scaling holds and the domain-growth process can be characterized, independently on $x$, by a unique length which evolves according to $l(t) \sim t^{n}$ being $n \sim(0.50-0.40)$. Although the growth process tends to be slower as $x$ increases, we find that the domain-wall motion follows the main assumptions underlying the Allen-Cahn theory. This is indicative that the coupling between diffusive excess particles and curvature-driven interface motion does not modify the essential time dependence but varies (slows down) the growth rate of the growth law, i.e., $l(t)=k_{x} t^{1 / 2}$, with $k_{x}$ decreasing with $x$. We suggest that the logarithmic growth experimentally observed in some nonstoichiometric binary materials has to do with the existence of specific interactions (not present in our case) between diffusive particles and domain walls. These interactions are of crucial importance in determining the essential time dependence of the growth law. [S0163-1829(96)05221-6]
\end{abstract}

\section{INTRODUCTION}

The problem of a system thermally quenched below a phase transition represents a prototypical nonequilibrium situation of great interest from both a theoretical and experimental point of view. ${ }^{1-5}$ In particular, understanding of the role of the randomness present in impure and imperfect systems defines one of the present challenges in statistical mechanics and material science and centers most of the recent investigations.

The present work deals with the kinetics of domain growth of a nonstoichiometric binary alloy $A_{x} B_{1-x}$ which undergoes an order-disorder transition. At a given temperature, the stable configurational ordered structure depends on $x$. Ordered structures are always defined in terms of a given specific value of the composition. For instance, in a bcc lattice, $x_{0}=0.50$ is associated with a $B 2$ structure and $x_{0}=0.75$ with a $\mathrm{DO}_{3}$. The composition associated with an ordered structure is called the stoichiometric composition $x_{0}$. Nonstoichiometric values of the composition lead to deviations from the, generally well-established, ideal behavior in both the statics and the dynamics.

When a binary alloy is deeply quenched inside the lowtemperature ordered phase, the new phase appears in the form of small domains separated by interfaces. The quantity of interest is the average size of the domains which grows with time according to a remarkable degree of universality. ${ }^{1,3}$ For nonstoichiometric binary alloys $\left(x \neq x_{0}\right)$, the excess particles, defined as the number of particles of the majority component (either of type $A$ or $B$ ) exceeding the stoichiometric composition, will have influence on both the bulk structure and the internal interfaces. In our case, those interfaces are antiphase boundaries (APB's), which are defined by variations in the long-range order parameter. A general characteristic encountered during the evolution towards the equilibrium in nonstoichiometric binary alloys is the tendency for the excess particles to accumulate at the interfaces. This effect, in some context called interfacial adsorption, is more pronounced at short times and gives rise to overordered growing domains. Only at late times are the excess particles expelled back to the bulk and equilibrium order inside the domains may be (asymptotically) reached. The problem of growing domains with local order transiently larger than the equilibrium values has been recently discussed in other situations such as diluted systems ${ }^{6}$ and multicomponent systems ${ }^{7}$ and suggested to be a generic effect in nonequilibrium ordering dynamics. ${ }^{8}$

From the extensive literature existing on the dynamics of ordering it is clearly established that experiments, ${ }^{9-14}$ theory, ${ }^{15}$ and computer simulations ${ }^{16-18}$ agree that, for stoichiometric binary alloys undergoing an order-disorder transition, the kinetics of domain growth can be character- 
ized by a unique length which evolves with time according to the Allen-Cahn growth law $l(t) \sim t^{1 / 2}$. It refers to systems with a nonconserved order parameter and the velocity of an element of the internal surface is proportional to its local curvature. ${ }^{15}$ Unfortunately much less is known for the case of nonstoichiometric alloys. There exist, however, a number of computer simulation studies on annealed vacancy effects $^{6,19,20}$ that predict precipitation of the vacancies at the domain walls. The corresponding dynamics is markedly slower, eventually leading to a complete pinning of the domain walls. In light of these results on vacancy effects it has been suggested ${ }^{19}$ that the problem of ordering kinetics in nonstoichiometric binary alloys is closely related to that of annealed vacancies or impurities. The existence of an accumulation of excess particles at the interfaces in binary alloys has been experimentally observed ${ }^{21,22}$ and predicted by computer simulations. ${ }^{23}$ Besides, it has been reported ${ }^{21}$ that the ordering kinetics in $\mathrm{Cu}_{0.79} \mathrm{Au}_{0.21}$ shows a crossover from the Allen-Cahn growth law, for stoichiometric $\mathrm{Cu}_{3} \mathrm{Au},{ }^{11}$ to a logarithmic growth law. This dramatic slowing down has been attributed to the formation of clusters of extra $\mathrm{Cu}$ atoms. ${ }^{21}$ These clusters have less mobility than a single atom and the dynamics can be interpreted in terms of an Ising system with quenched random impurities which is known to lead to a logarithmic growth behavior. ${ }^{24}$ It is worth mentioning that these randomly placed quenched impurities do not generate random fields. ${ }^{24} \mathrm{~A}$ different interpretation for experiments on $\mathrm{Cu}_{0.79} \mathrm{Au}_{0.21}$ is given in Ref. 19 in terms of the precipitation of excess $\mathrm{Cu}$ atoms at the domain walls which may cause the curvature-driven Allen-Cahn growth mechanism to become ineffective. We shall provide insight into this problem by showing that the effect of precipitation itself is not responsible for the slowing down of the dynamics.

In the present paper we report on a Monte Carlo study of the influence of adsorbed excess atoms on the migration kinetics of APB's in nonstoichiometric binary alloys and its effect on domain-growth kinetics. We use a two-dimensional antiferromagnetic Ising model with nonconserved order parameter. The model accounts for the adsorption of excess atoms at the APB's which ultimately may lead, provided the temperature is low enough, to a complete saturation of the interfaces. In general, the growth of the domains can be studied using two different quantities: the excess energy and the structure factor. It turns out that when precipitation at the domain walls is present $\left(x \neq x_{0}=0.50\right)$ the excess energy is not a suitable measure for the average domain size. ${ }^{8,19,25}$ In this last case, it is interesting to know the total perimeter of the nonequilibrium interfaces. The analysis of the data obtained from the time evolution of the total interface length and from the structure factor allows us to conclude that during the saturated regime and independently of $x$ scaling holds and the ordering process can be characterized by a unique length which evolves according to a power law consistent with the Allen-Cahn growth law. Furthermore, a carefull analysis of the movement of the interfaces seems to indicate that they evolve by covering a constant domain area per unit of time. This feature is of key importance in the Allen-Cahn theory. ${ }^{17,26-28}$

We now put forward an outline of our main conclusions. The existence of adsorption of the excess particles at the interfaces is dictated by energetic reasons and should be en- visaged as a transient state the system attains as the easiest way to rapidly decrease the free energy by decreasing the internal energy. The subsequent evolution towards equilibrium necessarily implies the elimination of the interfaces. This requires the ejection of excess particles from the interfaces and its dissolution inside the bulk with the corresponding increase of the mixing entropy. This subtle interplay between internal energy and mixing entropy was first pointed out by Gilhøj et al. ${ }^{6,8}$ who suggested it is a generic effect in nonequilibrium dynamics. Furthermore, the diffusive motion of the excess particles inside the domains couples to the migrating saturated interfaces. Nevertheless, since in our case there is no specific interaction between diffusive particles and saturated interfaces, this coupling does not modify the essential time Allen-Cahn dependence but introduces a functionality on the composition in the growth rate of the growth law. In the discussion (Sec. VI) we suggest that a specific interaction between diffusing extra matter and the saturated interfaces (not present in our case) is the physical origin of the slow (logarithmic) domain growth behavior observed in $\mathrm{Cu}_{0.79} \mathrm{Au}_{0.21}{ }^{21}$

The outline of the paper is the following. In Sec. II we briefly describe the model Hamiltonian and its associated microdynamics including some relevant computational details. In Sec. III we analyze the Monte Carlo results obtained for the time evolution of the excess internal energy and show that it is not a suitable measure for the study of the domaingrowth process. Next, in Sec. IV, the same study is done again but now in terms of the time evolution of the total perimeter of the interface. This is complemented with the evolution of the excess particles obtained by tracking them all along the ordering process. In Sec. V we present the Monte Carlo results obtained from a systematic study, as a function of $x$, of the domain-growth process using two measures for the growth: the interface perimeter and the second moment of the structure factor. Finally, in Sec. VI we discuss the results and provide our conclusions.

\section{MODEL DYNAMICS}

The binary alloy $A_{x} B_{1-x}$ is modeled by a twodimensional antiferromagnetic Ising model defined on a square lattice $(L \times L=N)$ subjected to periodic boundary conditions. The reason for restricting the present study to two dimensions is because the topology of the domain configurations can be easily visualized and thus analyzed. The corresponding Hamiltonian is

$$
\mathscr{H}=J \sum_{\mathrm{NN}} S_{i} S_{j}+\mathscr{H}_{0}(x)
$$

where $S_{i}= \pm 1$ and $J>0$. The summation extends over all nearest-neighbor (NN) pairs. The composition is defined as $x=N_{A} / N, N_{A}$ being the number of $A$ particles and $N_{B}=N-N_{A}$ the number of $B$ particles. The term $\mathscr{H}_{0}(x)$ depends on composition and will be taken as constant. ${ }^{29}$

The set of variables $\left\{S_{i}\right\}$ is updated according to the Kawasaki (spin-exchange) dynamics implemented into the Metropolis Monte Carlo algorithm. ${ }^{30-32}$ By this procedure the summation $\Sigma_{i} S_{i}$ and therefore the composition $x$ are conserved $\left[\Sigma_{i} S_{i}=N_{A}-N_{B}=N(2 x-1)\right]$ whereas the order pa- 


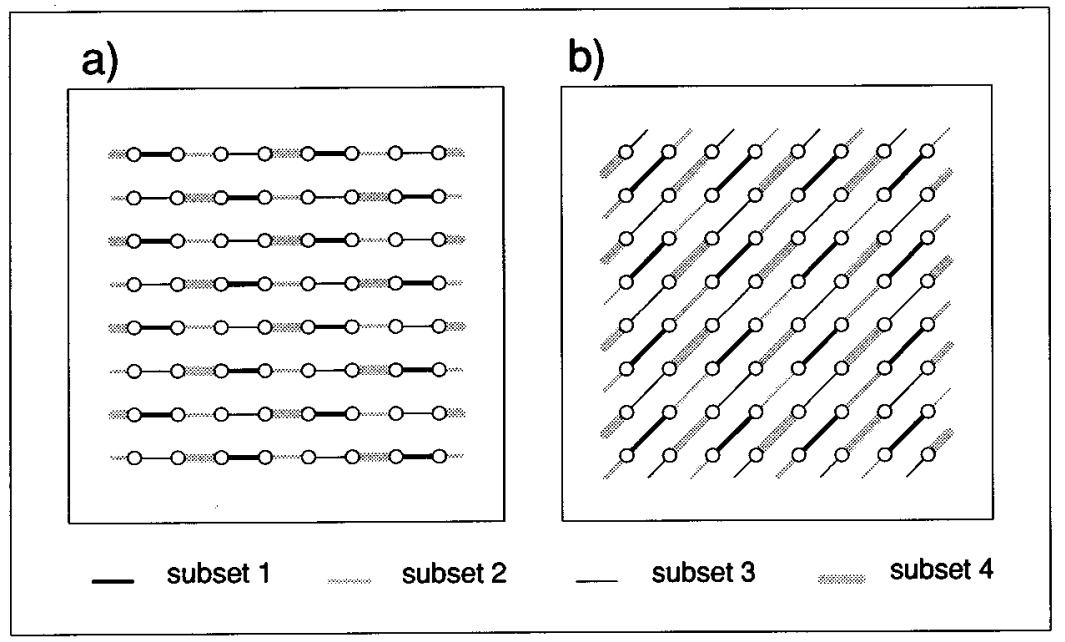

FIG. 1. Representation of the different subsets in which the set of all possible NN (a) and NNN (b) exchanges is subdivided in the parallel updating. In the figures only the horizontal $\mathrm{NN}$ exchanges and one of the two diagonal NNN exchanges are represented. By rotating both pictures $90^{\circ}$ one gets the vertical $\mathrm{NN}$ exchanges and the other diagonal NNN exchanges, respectively.

rameter (the total antiferromagnetic order) is not. To avoid low-temperature freezing-in behavior ${ }^{18,33}$ both exchanges between NN and next-nearest-neighbor (NNN) particles are allowed. The initial configuration is prepared randomly (very high temperature) and the time scale is given in units of attempted Monte Carlo particle exchanges per site (MCS). In a square lattice, the four different kinds of allowed exchanges are the following: NN horizontal exchange, NN vertical exchange, and two NNN diagonal exchanges. For each one, the set of all possible exchanges of that kind is subdivided into four subsets (see Fig. 1). In this way, the different pairs of particles to be exchanged within a subset have no direct interaction bonds between them, which allows for parallel updating. The 16 different subsets are periodically chosen at random and all exchanges within the selected subset are attempted sequentially.

\section{EVOLUTION OF THE EXCESS INTERNAL ENERGY}

The ordering process, governed by the model described in Sec. II, is studied as a function of time $t$ in response to a deep quench from very high to a low temperature $T=0.25 \mathrm{~J} / K_{B}$, well inside the ordered region.

We start by studying the time evolution of the excess internal energy per particle, defined as

$$
\Delta E(t)=E(t)-E(T),
$$

where $E(t)$ is the nonequilibrium energy and $E(T)$ is the energy at the quench temperature $T$, calculated in equilibrium conditions.

The reason behind the use of $\Delta E(t)$ as a measure of the linear length scale associated with the growth of the domains is that during the coarsening regime, the total excess internal energy is mainly contained in the domain walls (the bulk is in equilibrium) and therefore $\Delta E(t)$ can be directly related to the total perimeter of the nonequilibrium APB's. Then, provided scaling holds, $\Delta E(t)$ is a measure of the inverse of the average linear domain size. ${ }^{34} \mathrm{We}$ shall see below that this does not apply in nonstoichiometric binary alloys.

In Fig. 2 we show the time evolution of the total excess internal energy for the alloys $x=0.50,0.51,0.52$, and 0.53 for different lattice sizes. The averages are performed over ten independent runs. The results clearly show that the ex-

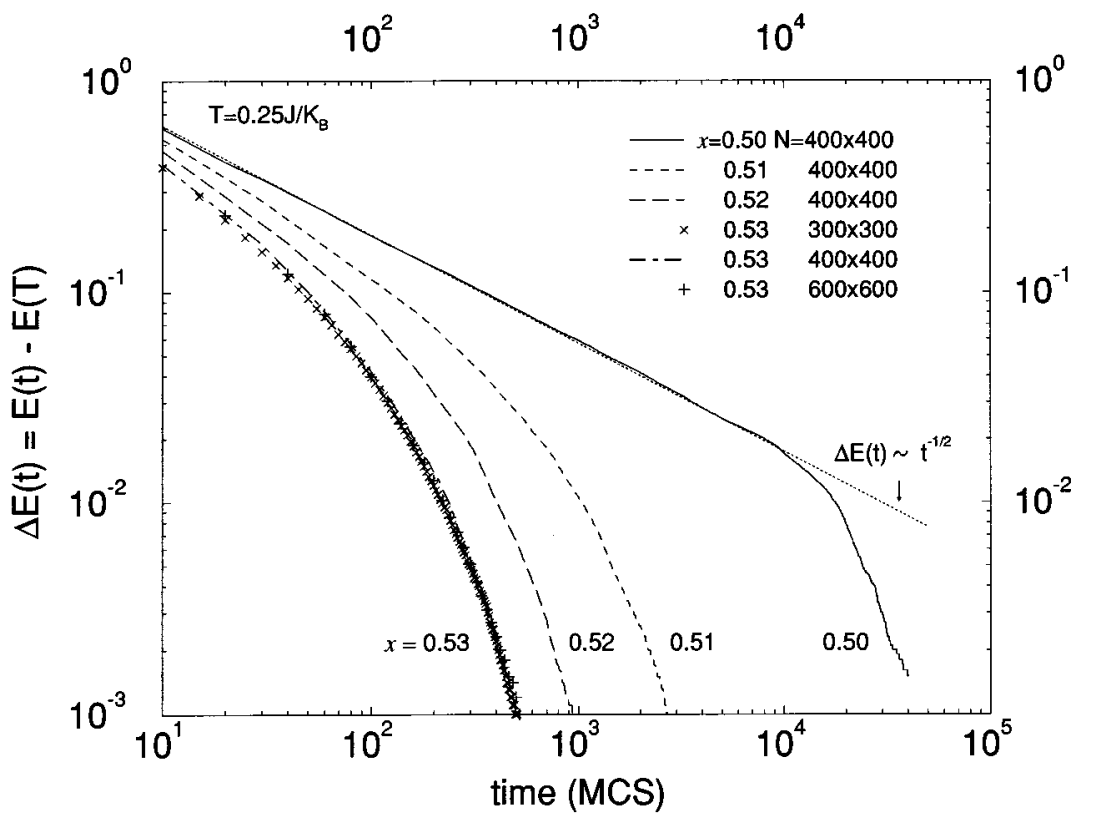

FIG. 2. Log-log plot of the excess internal energy $\Delta E(t)$, in units of $J$, versus time for different values of the composition and different lattice sizes. The power-law behavior for $\Delta E(t)$ at $x=0.50$ is emphasized with a straight dotted line. The quench temperature is in all cases $T=0.25 J / K_{B}$. 

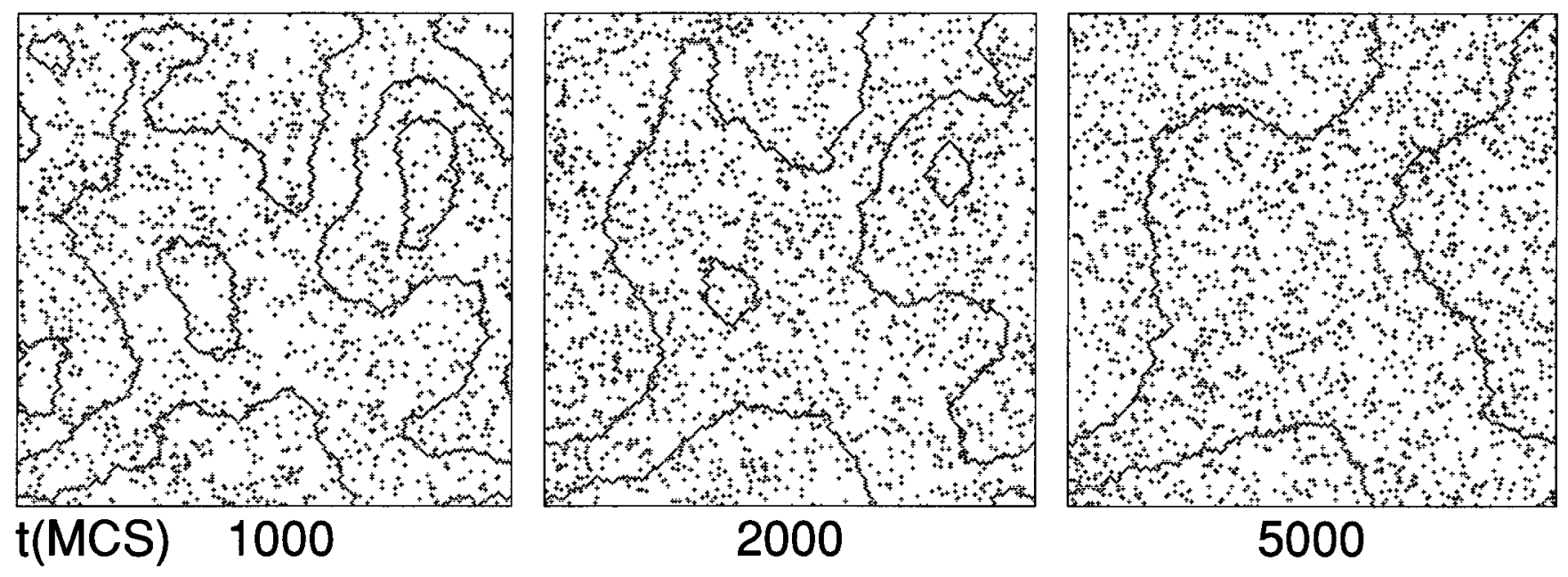

FIG. 3. Snapshots of the evolving domain structure for the case $x=0.53$ and $N=300 \times 300$. Figure 2 shows the corresponding time evolution for the excess internal energy. Black areas represent disordered particles whose energy is different from $-4 J$.

cess energy (2.1) does not follow a power law as happens in the stoichiometric case $\left(x=x_{0}=0.50\right)$. After a period of time which is independent of the lattice size, the internal energy of the system $E(t)$ reaches a value which turns out to be equal to the equilibrium one so that the excess internal energy is, for longer times, $\Delta E(t)=0$. This is because a nonstoichiometric alloy with only $\mathrm{NN}$ interactions may create APB's with no change in the internal energy. ${ }^{35}$ We stress that in spite of the fact that $\Delta E(t)=0$, the system is not in equilibrium yet (there are still interfaces) and the ordering process spontaneously proceeds in order to reach the thermodynamic equilibrium, as can be seen from some selected snapshots of the domain structure (Fig. 3). From these results it then follows that the excess of internal energy is not proportional to the total perimeter of the interfaces. This happens to be a quite general feature not only for nonstoichiometric binary alloys but as well for the cases of diffusing impurities ${ }^{25}$ and diluted systems. ${ }^{19}$ It is then desirable to obtain the time evolution of the total perimeter of the interface length in order to have a direct suitable measure for the domain-growth process. This will be done in the next section by studying the time evolution of the distribution of excess particles.

\section{EXCESS-PARTICLE DISTRIBUTION AND INTERFACE PERIMETER}

The excess particles, defined in terms of the majority component as $N_{e}=N_{A}-N / 2$ (if $N_{A}>N_{B}$ ), can be found either inside the bulk or located at the interfaces.

For our purposes, a nonstoichiometric binary alloy can be regarded as an ideal stoichiometric two-component system having two kinds of imperfections: excess particles, immersed in a perfectly ordered bulk, and interfaces. To calculate the energy associated with those imperfections we take the ground state (perfectly ordered) of the stoichiometric system as the state of reference. By subtracting the energy of such a state of reference to the actual internal energy of the nonstoichiometric binary alloy we obtain the excess energy associated with the imperfections. Next, one needs to dis- criminate which part of this excess internal energy is due to the bulk excess particles and which part is due to the interfaces. By doing this we may evaluate the total length associated with the entire interface network. Notice that this reference state is not the equilibrium state of the nonstoichiometric binary alloy and has only been defined to compute the total interface length.

The excess particles inside the bulk have an interaction energy of $4 \mathrm{~J}$. Moreover, there will always be some disorder present inside the bulk caused by thermal fluctuations or induced by the quench. The corresponding disordered particles have an energy of $4 J$ as well. Assuming that the number of $A$ particles with energy $4 J$ associated with disorder is equal to that of $B$ particles, we can estimate the total number of excess particles inside the bulk as $N_{e}^{b}=\Sigma_{\left(i \in\left\{N_{4}\right\}\right.} S_{i}$, where $\left\{N_{4}\right\}$ is the set of particles with energy $4 J$. Thus, the excess of internal energy with respect to the reference state due to the excess particles inside the bulk is $8 N_{e}^{b}$.

Regarding the interface, it can be seen as a network of equal-particle $(A-A$ or $B-B)$ bonds. Thus, the excess internal energy with respect to the reference state due to the interface is $2 J$ per bond. If we measure the length of the interface in terms of the number of these bonds, the entire interface length $I$ can be obtained from the expression

$$
\mathscr{H}-(-2 N J)=8 N_{4} J+2 I J,
$$

where $N_{4}$ is the number of particles with energy $4 J$. In order to account for the disorder, we have taken $8 N_{4} J$ as the excess energy associated with the bulk rather than $8 N_{e}^{b} J$.

It is very instructive to analyze the evolution of the density of excess particles at the interface, $\lambda$, defined as

$$
\lambda=\frac{1}{I}\left(N_{e}-N_{e}^{b}\right)
$$

In Fig. 4 we simultaneously display the time evolution for the interface length $I$ (log-log scale) and for the density of excess particles at the interface $\lambda$ (log-linear scale). The parameters of the simulated system are $N=600 \times 600$ and 


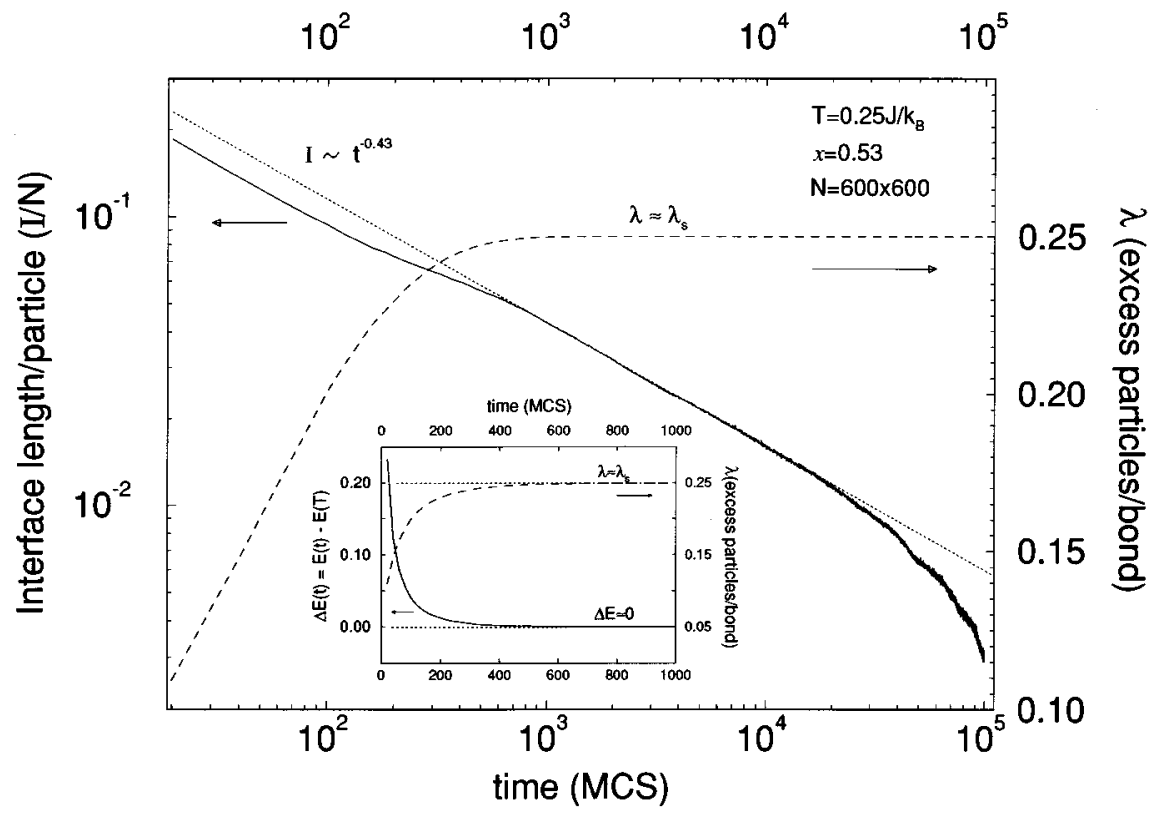

FIG. 4. Log-log plot for the time evolution of the total interface length per particle, $I / N$ (solid line) for $x=0.53, T=0.25 \mathrm{~J} / K_{B}$, and $N=600 \times 600$. We simultaneously display, in a semilogarithmic scale, the corresponding evolution for the density of excess particles at the evolving interface, $\lambda$ (dashed line). Notice that after the saturation of the interface, its decrease follows an algebraic behavior which is emphasized by the dotted line. In the inset we show the correlation between $\lambda$ (dashed line) and the excess internal energy $\Delta E$ (solid line).

$x=0.53$. The results reveal the existence of two differentiated regimes. During the initial transient regime the density of excess particles at the interface increases rapidly with time, indicating that the interfaces act as an attractor for bulk excess particles (in other words, the excess particles precipitate at the APB's) until it reaches a saturated value, $\lambda_{s}$. During the second regime, which we call the saturated regime, the density of excess particles at the interface remains constant, $\lambda \sim \lambda_{s}$, and the interface perimeter $I$ decreases following a power-law behavior. This is indicative of an algebraic behavior of the type $I \sim t^{-n}$. The main point is that although $\lambda$ remains constant during the saturated regime, the number of excess particles in the bulk must increase as a consequence of the shrinking of the interfaces. Consequently, the composition in the bulk, $x_{b}$, increases with time and tends asymptotically to the equilibrium value $x$. Actually, $x_{b}$ remains, for finite times, below $x$ and therefore the internal energy per particle in the bulk is lower than its equilibrium value. This is the reason why the excess internal energy cannot be directly related (is not proportional) to the total perimeter of the interface and consequently is not a good measure for the average size of the domains. In the inset of Fig. 4 we show the corresponding time evolution for the excess internal energy. Notice that during the saturated regime the internal energy of the system has the equilibrium value. We shall return to this point below.

The behavior shown in Fig. 4 is intrinsically related to the process of interfacial adsorption, i.e., the adsorption of excess particles by the interface, which is dominant at very short times. The process of adsorption involves the exchange (NNN exchange) of excess particles in the bulk (in our case of type $A$ ) by particles of the other species $(B)$ located at the interface. Notice that by this mechanism the system decreases its internal energy in an amount of 4, 8, or 12 (in units of $J$ ), depending on the neighborhood of the $B$ particle at the interface. In any case, the internal energy of the alloy decreases. Thus, the process of adsorption of excess particles by the interface is dictated by energetic reasons in response to the principle of reducing free energy. At early times, this can be easily accomplished by means of local rearrangements which rapidly decrease the internal energy. However, this spontaneous process finishes when there are no excess particles left in the bulk or when all bonds at the interface are $A-A$ bonds (bonds of excess particles). In this last case the number of $A$ excess particles adsorbed per unity of interface can be evaluated and is found to be $\lambda_{s}=0.25$ particles/bond (see Appendix A). This is what we call the saturation density, denoting that the interface cannot adsorb more excess particles. The value $\lambda_{s}=0.25$ refers to a complete saturated interface and is exact at $T=0 \mathrm{~K}$. As the temperature is raised the maximum concentration of excess particles at the interface does not correspond to complete saturation but is smaller than $\lambda_{s}$. This can be explained in the following way. The behavior of $N_{e}$ can be envisaged as that of a two-level energy system. At $T=0 \mathrm{~K}$, the particles tend to be all confined in the low-energy level, i.e., the interface, giving rise to complete saturation provided the total number of excess atoms $N_{e}$ and the interface length are appropriated. As the temperature is increased, thermal fluctuations provide the mechanism for the excess particles to gradually jump up to the high-energy level (the bulk). As a consequence of this, the maximum concentration of excess particles at the interface will be lower than $\lambda_{s}$. In our simulations, all the quench experiments are performed at very low temperature $\left(T=0.25 \mathrm{~J} / K_{B}\right)$ and the maximum concentration of excess particles at the interface is very close to $\lambda_{s}$ as can be seen in Fig. 4. We have performed a few simulations at higher temperature and obtain the same qualitative behavior as in Fig. 4 in the sense that there exists a regime where the interface evolves at constant $\lambda$, although this value is lower than the one at $T=0 \mathrm{~K}, \lambda_{s}$. The dynamics as a function of the temperature will be studied elsewhere.

An important consequence of this saturation phenomenon discussed above and present in our low-temperature simulation is that the value of the corresponding internal energy coincides with that of the equilibrium (see the inset of Fig. 4). This can be understood by simple energetic consider- 
ations. One unit of interface raises, independently of the excess particles, in 2 units the internal energy of the system with respect to the reference state, whereas it means 8 units for each excess particle in the bulk. Thus, 4 units of interface length mean the same excess energy to the system as one excess particle in the bulk. Then if the interface is saturated, each 4 units of interface contains an excess particle ( $\lambda_{s}=0.25$ particles/bond) and there is no change in the internal energy of the alloy if the interface is shrunk in 4 units and the excess particle they contain is ejected to the bulk. This property was first discussed in a very general context by Cahn and Kikuchi ${ }^{35}$ and can be summarized as follows: The excess of internal energy in a nonstoichiometric binary alloy due to a saturated interface is exactly compensated by the energy flow in the bulk due to the adsorption of excess particles by the interface.

We end this section by outlining the overall picture which emerges from the whole set of data presented above. Immediately after the quench, the new phase appears in the form of very small domains separated by internal interfaces (APB's). Once these are formed, the principle of reducing free energy is easily achieved by means of local rearrangements which rapidly reduce the internal energy. These rearrangements consist in exchanges of excess particles (of the majority component) in the bulk by particles (of the minority component) at the interface. In this way the system localizes as much disorder as possible at the interfaces. This process follows on until the interface saturates. Henceforth, the interface will evolve under this saturated regime (or regime of $\lambda \sim$ const) and the subsequent relaxation proceeds by means of eliminating interfaces. This elimination process requires the ejection of the excess particles towards the bulk which, in turn, makes the composition asymptotically to approach to the nominal equilibrium value. During this second regime the system remains in its equilibrium internal energy and the free energy is balanced by maximizing the entropy. We find that during the saturated regime, the behavior of $I$ follows a power law $I \sim t^{-n}$. This is indicative that the average domain size $l$ grows according to a power law characterized by an exponent $n, l(t) \sim t^{n}$. In order to evaluate the growth exponent $n$ we have performed extensive calculations for different values of the composition $x$ and for different system sizes. These will be presented in the next section.

\section{DOMAIN-GROWTH KINETICS}

\section{A. Interface perimeter}

In order to obtain a measure for the time evolution of the average domain size, we begin by studying the time evolution of the entire interface length $I$. The information of interest collected from every configuration is the energy distribution of particles. From this we calculate $I$ by using expression (4.1).

The simulations have been carried out for three different values of the composition $x=0.51,0.52$, and 0.53 . For larger values of $x$, the fluctuations make the use of expression (4.1) doubtful. In order to minimize finite-size effects, we have studied different sizes of the lattice $(N=300 \times 300$, $N=400 \times 400$, and $N=600 \times 600$ ). The results have been averaged over ten different realizations using different initial random (disordered) configurations. For $x=0.51$, the number
TABLE I. Growth exponent $n$ obtained from the total perimeter length $\left(I \sim t^{-n}\right)$.

\begin{tabular}{lcc}
\hline \hline$n$ & $x=0.52$ & $x=0.53$ \\
\hline$L=300$ & $0.40 \pm 0.12$ & $0.40 \pm 0.12$ \\
$L=400$ & $0.44 \pm 0.06$ & $0.39 \pm 0.05$ \\
$L=600$ & $0.44 \pm 0.05$ & $0.43 \pm 0.05$ \\
$L \rightarrow \infty$ & $0.5 \pm 0.1$ & $0.45 \pm 0.1$ \\
\hline \hline
\end{tabular}

of excess atoms turns out to be not enough to rapidly saturate the interface. Only later on, already in the domain-growth regime, when the total length of the interface has diminished conveniently, may the system reach the saturated regime. Unfortunately, even for the largest system $(600 \times 600)$, finite-size effects appear before such a regime can be clearly seen. For the other two compositions, $x=0.52$ and $x=0.53$, there exists an interval of time between the saturated regime and the apparition of finite-size effects which allows for an accurate evaluation of the growth exponent. In Table I we show the values of the exponent $n$ obtained by fitting the expression $I \sim t^{-n}$ to the Monte Carlo data.

The main result is that, during the saturated regime, the ordering evolution proceeds, independently of $x$, in an algebraic manner. We do not observe, at least for the values of $x$ studied here, any correlation between the exponent $n$ and the value of $x$. Although $n$ remains, in all cases, slightly below $1 / 2$, the results are consistent with the curvaturedriven Allen-Cahn exponent, ${ }^{15}$ characteristic of a system with a nonconserved order parameter. However, it is not obvious that the basic assumptions underlying the Allen-Cahn theory apply for saturated interfaces. In addition, it has been suggested $^{19}$ that the diffusional motions of the excess atoms in the bulk couple to the migrating interfaces and should therefore interfere with the dynamics. More results are necessary in order to elucidate more about this point. We would like to mention that in spite of the fact that the data obtained from the analysis of the interface length are not definitive, the study itself is very instructive and provides a picture very much on the line of recent assertions concerning the existence of overshooting effects in nonequilibrium ordering dynamics. $^{6-8}$

In order to gain in understanding it is imperative to extend the calculations to larger values of $x$. Given that the use of expressions (4.1) and (4.2) is limited to the range of small fluctuations of either temperature and/or concentration, we shall perform structure factor calculations. In addition these will provide information about the dynamical scaling properties.

\section{B. Structure factor and dynamical scaling}

The structure factor provides an overall description of the ordering process. Assuming translational invariance, it is given by

$$
S(\vec{k}, t)=\frac{1}{N}\left\langle\left|\sum_{j} e^{i \vec{k} \cdot \vec{r}_{j}} S_{j}(t)\right|^{2}\right\rangle,
$$

where $\vec{r}_{j}$ are the positions of all the lattice sites and $\vec{k}$ is a vector of the reciprocal lattice. For an $N=L \times L$ square lat- 


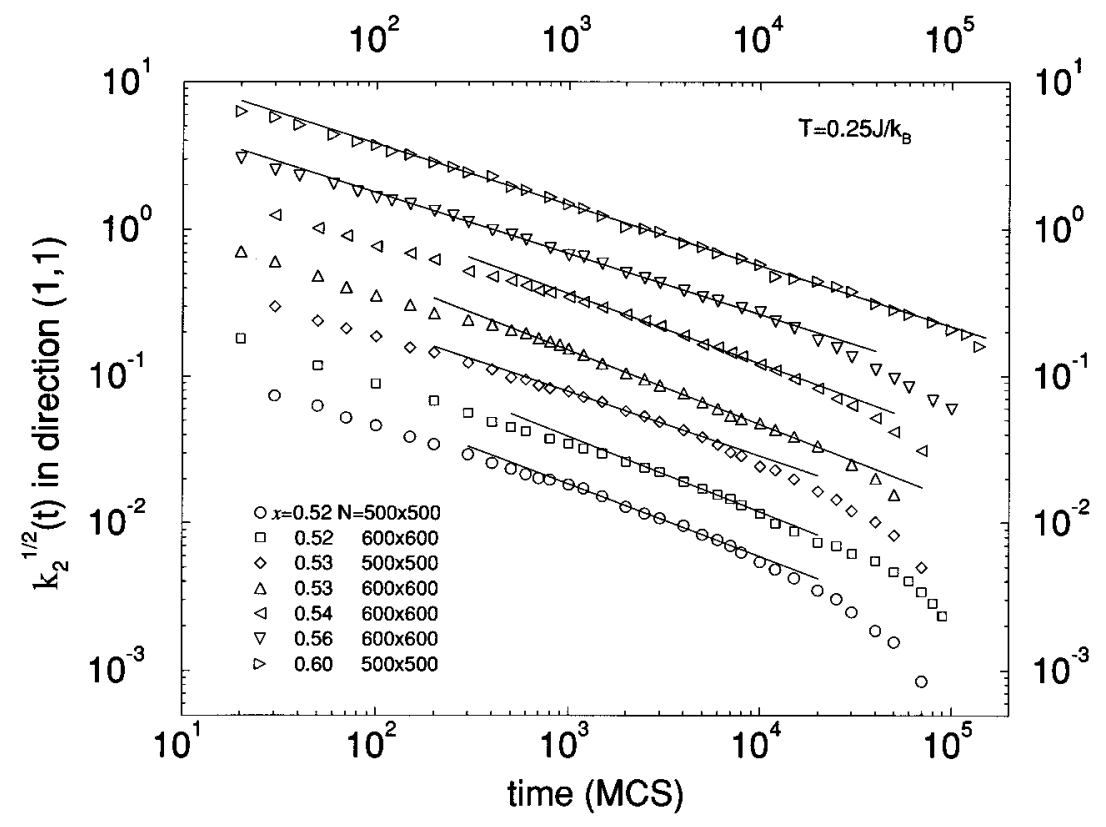

FIG. 5. Log-log plot of the square root of the second moment of the structure factor $k_{2}^{1 / 2}(t)$ versus time for different values of the composition $x$ at the quench temperature $T=0.25 J / K_{B}$. The bottom set of data corresponds to units on the $y$ axis whereas each of the other data sets is shifted a factor of 2 above the one below. The solid lines are guides to the eye.

tice with periodic boundary conditions, $\vec{k}$ can take the values $\vec{k}=(n, m)(2 \pi / L a)$, where $a$ is the lattice parameter and $n$ and $m$ are integers. Due to the antiferromagnetic order, a peak develops around $\vec{k}=(\pi / a)(1,1)$. We have calculated the structure factor in the directions $(1,0)$ and $(1,1)$ of the reciprocal lattice. The results are an average over the symmetrical directions $(0,1)$ and $(1,-1)$, respectively, and over ten independent runs. The mean size of the domains is obtained from the second moment of the structure factor, $k_{2}(t)$, using

$$
l(t) \sim k_{2}^{-1 / 2}(t)
$$

where

$$
k_{2}(t)=\frac{\sum_{\vec{k}=(\pi / a)(1,1)}^{\vec{k}_{\max }}|\vec{k}-(\pi / a)(1,1)|^{2} S(\vec{k}, t)}{\sum_{\vec{k}=(\pi / a)(1,1)} S(\vec{k}, t)} .
$$

The summations in Eq. (5.3) are over all possible values of $\vec{k}$ along the corresponding direction and are cut when $S(\vec{k}, t)$ is less than the background values obtained for a completely disordered configuration.

We have performed simulations for values of the composition $x=0.52,0.53,0.54,0.56$, and 0.60 and for two different values of the lattice size $N=500 \times 500$ sites and/or $N=600 \times 600$ sites. In Fig. 5 we show the time dependence for the square root of the second moment of the structure factor, $k_{2}(t)$, for different values of the composition $0.52 \leqslant x \leqslant 0.60$. We obtain that $k_{2}^{1 / 2}(t)$ evolves with time according to a power law with an exponent $n \sim 0.52-0.40$ consistent with the Allen-Cahn exponent. The results are summarized in Table II. It can be observed that for $x=0.52,0.53$, and 0.54 the exponent is remarkably close to $n=1 / 2$, while for the two largest values of the composition studied, $x=0.56$ and $0.60, n$ remains slightly below the Allen-Cahn exponent. This may be due to the enhancement of the fluctuations of concentration as $x$ increases which, in turn, increases the concentration of diffusing impurities. Actually, we have obtained that the equilibrium long-range order parameter rapidly drops down as we increase $x$ over $x=0.60$, being exactly zero at $x \geqslant 0.64 \pm 0.01$. Thus, for larger values of $x>0.60$ the ordering kinetics will be described by an effective exponent affected by large fluctuations of the order parameter associated with a phase transition located at $x=0.64$ for $T=0.25 \mathrm{~J} / K_{B}$.

From the shape of the structure factor at different times, we have tested the existence of statistical self-similarity during the domain-growth process. Figure 6 shows the scaling function

$$
k_{2}(t) S\left(\left|\vec{k}-\frac{\pi}{a}(1,1)\right| k_{2}^{-1 / 2}(t), t\right)
$$

in a semilogarithmic plot for the case $x=0.53$ and

\begin{tabular}{|c|c|c|c|c|c|c|}
\hline \multicolumn{2}{|l|}{$n$} & \multirow{2}{*}{$\begin{array}{c}x=0.52 \\
0.54\end{array}$} & \multirow{2}{*}{$\begin{array}{c}x=0.53 \\
0.44\end{array}$} & \multirow[t]{2}{*}{$x=0.54$} & \multirow[t]{2}{*}{$x=0.56$} & \multirow{2}{*}{$\begin{array}{c}x=0.60 \\
0.37\end{array}$} \\
\hline Direction $(1,0)$ & $L=500$ & & & & & \\
\hline & $L=600$ & 0.56 & 0.50 & 0.47 & 0.39 & \\
\hline \multirow[t]{2}{*}{ Direction $(1,1)$} & $L=500$ & 0.49 & 0.44 & & & 0.41 \\
\hline & $L=600$ & 0.52 & 0.51 & 0.48 & 0.41 & \\
\hline \multicolumn{2}{|c|}{ Mean value of $n$} & 0.52 & 0.47 & 0.475 & 0.40 & 0.39 \\
\hline
\end{tabular}
$N=600 \times 600 .^{36}$ The structure factor at all different times is

TABLE II. Growth exponent $n$ obtained from the second moment of the structure factor $\left(k_{2}^{1 / 2} \sim t^{-n}\right)$. 


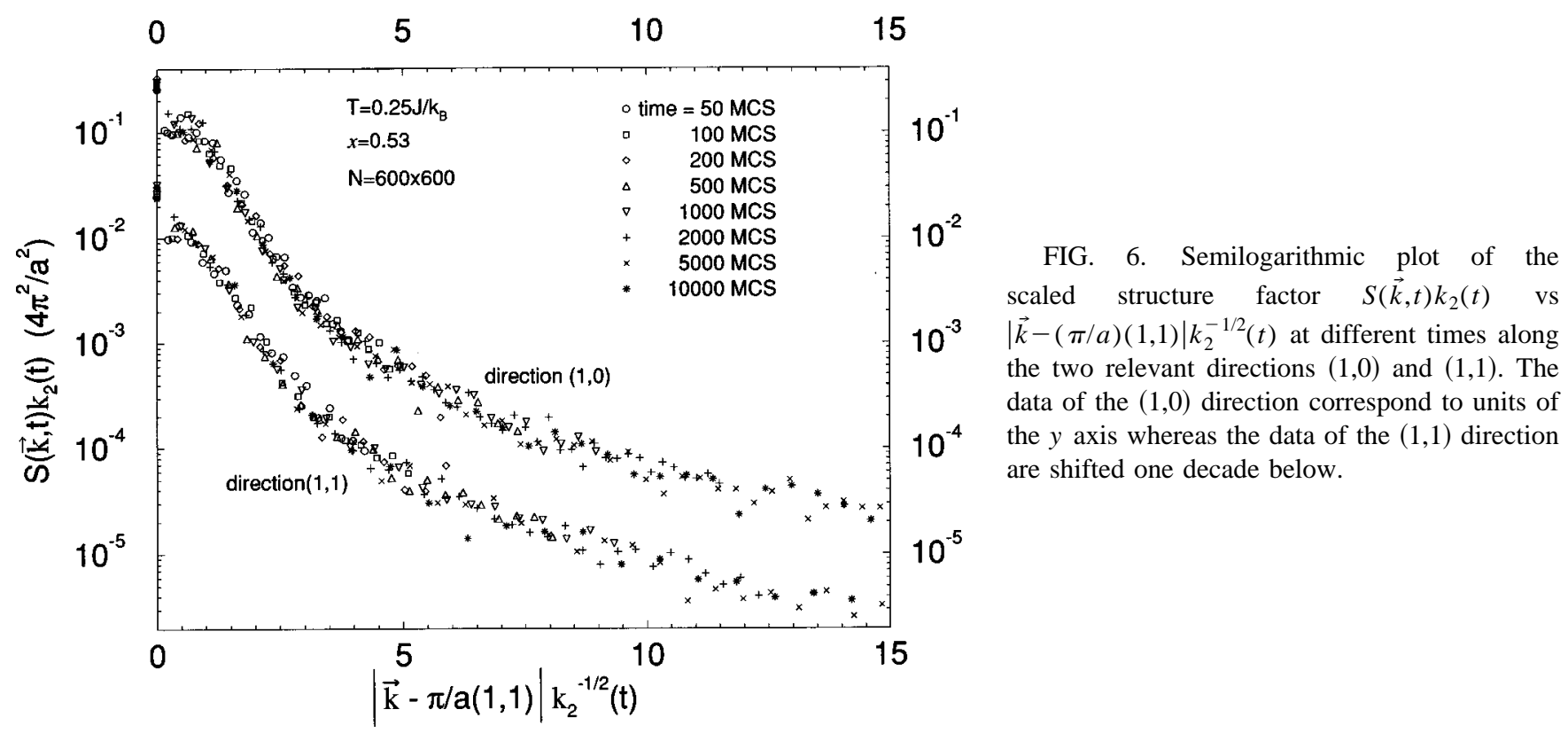

plotted only until $\vec{k}=\vec{k}_{\max }$. Although there are statistical fluctuations, the overlapping of the different curves is very satisfactory and ensures that the ordering process obeys dynamical scaling. Furthermore, the two measures of the growth yield the same time dependence for the growth law:

$$
I^{-1}(t) \sim k_{2}^{-1 / 2} \sim l(t)=\mathbf{K} t^{n} .
$$

The prefactor $\mathbf{K}$ is the growth rate and $t^{n}$ the essential time dependence. We evaluate $n=0.50 \pm 0.07$.

To elucidate more about the tendency shown by $n$ to apparently decrease as $x$ increases we have followed the evolution of single domains directly extracted from our simulations and monitored the domain area as a function of time. This is shown in Fig. 7 for two different values of the composition, $x=0.53$ and 0.56 . In both cases we obtain that the saturated interface evolves by covering a constant domain area per unit of time. This is accepted ${ }^{17,26-28}$ to be a feature of key importance in the Allen-Cahn theory.

One generally expects the presence of mobile impurities to provoke modifications of the growth law with respect to the pure ideal system. This change may be contained either in the growth rate and/or in the essential time dependence. In particular, it has been suggested that the coupling between the diffusive motion of the impurities and the motion of the domain interfaces should show up in the growth rate rather than in the essential time dependence. ${ }^{37}$

Following this, we have evaluated the growth rate from the structure factor $C$ defined by $k_{2}^{-1 / 2}=C t^{1 / 2}$, where $C$ and the growth rate $\mathbf{K}$, defined by $l(t)=\mathbf{K} t^{n}$, are related by a geometric factor, presumably independent of the composition. The results are shown in Table III.

We obtain a continuous smooth decreasing of $C$ as $x$ increases. Given that the characteristics of the interfaces are

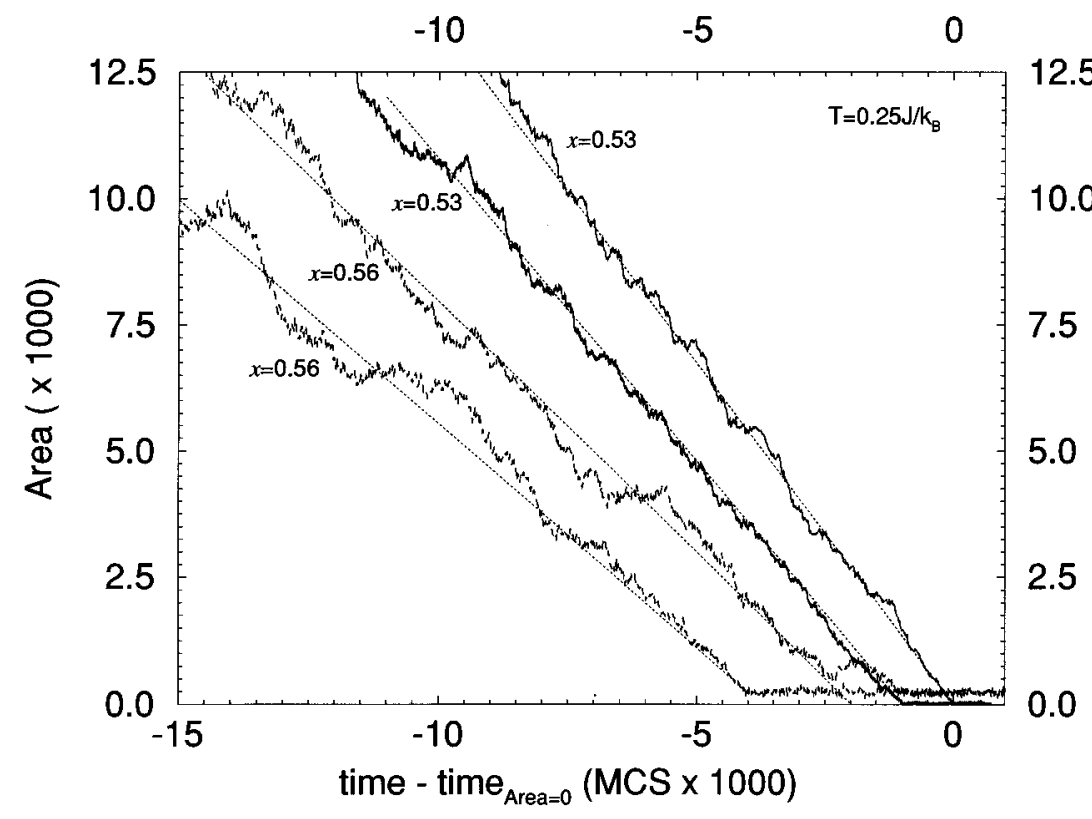

FIG. 7. The decrease in area for different single domains extracted from our simulations is approximately linear in time. Two processes correspond to $x=0.53$ (solid line) and the other two at $x=0.56$ (dashed line). The different sets of data are shifted along the $x$ axis in order to clarify the picture. The dotted lines are guides to the eye and denote the average linear behavior. 
TABLE III. Values of the prefactor $C$ obtained from the second moment of the structure factor $\left(k_{2}^{-1 / 2}=C t^{1 / 2}\right)$.

\begin{tabular}{|c|c|c|c|c|c|c|c|}
\hline \multicolumn{2}{|c|}{$C \quad\left[(\mathrm{MCS})^{-1 / 2} a / \pi\right]$} & \multirow{2}{*}{$\frac{x=0.50}{2.13}$} & \multirow[t]{2}{*}{$x=0.52$} & \multirow[t]{2}{*}{$x=0.53$} & \multirow[t]{2}{*}{$x=0.54$} & \multirow[t]{2}{*}{$x=0.56$} & \multirow[t]{2}{*}{$x=0.60$} \\
\hline & $L=400$ & & & & & & \\
\hline \multirow[t]{3}{*}{ Direction $(1,0)$} & $L=500$ & & 1.79 & 1.54 & & & 1.00 \\
\hline & $L=600$ & & 1.69 & 1.59 & 1.32 & 1.23 & \\
\hline & $L=400$ & 2.17 & & & & & \\
\hline \multirow[t]{2}{*}{ Direction $(1,1)$} & $L=500$ & & 1.69 & 1.49 & & & 1.01 \\
\hline & $L=600$ & & 1.69 & 1.69 & 1.33 & 1.28 & \\
\hline \multicolumn{2}{|c|}{ Mean value of $C$} & 2.15 & 1.72 & 1.58 & 1.32 & 1.26 & 1.01 \\
\hline
\end{tabular}

the same for all values of $x$ (saturated with the same value of $\lambda \simeq \lambda_{s}$ ), we attribute this slowing down of the growth rate to the enhancement of the concentration fluctuations as $x$ increases. These fluctuations generate small clusters of excess particles which interfere with the movement of the interfaces, yielding a slower growth rate but keeping unaltered the essential time dependence. Actually, we have verified that the evolution of a saturated interface is dictated by the curvature so that the domain area swept by the interface becomes linear with time. Consequently, the driving force is provided by the curvature and the ordering kinetics can be described in terms of a growth law with a time dependence of the Allen-Cahn type, $l(t) \sim t^{1 / 2}$. The effect of the diffusional motion of the excess atoms on the migration of the domain walls is contained on the growth rate $\mathbf{K}$ of the growth law which decreases as $x$ increases as a demonstration of the high importance of the interplay between mobile excess atoms and migrating interfaces. Notice that, once the interfaces saturate, this interplay does not take place via any specific interaction between diffusing excess atoms and interfaces. We suggest that this point is of crucial importance in determining the essential time dependence of the growth law.

\section{DISCUSSION AND CONCLUSIONS}

We use a nearest-neighbor antiferromagnetic Ising model with spin-exchange dynamics to study by Monte Carlo simulation techniques the ordering process in low-temperature quenched nonstoichiometric binary alloys $A_{x} B_{1-x}$. The main characteristic feature of this system is the tendency of the excess particles to precipitate at the domain interfaces.

In our case, we find that the accumulation of excess particles at the APB's initially increases very rapidly and, since the temperature is very low, it ultimately leads to a complete saturation of the interfaces. This saturated regime is available at finite computer times only for values of the composition $x \geqslant 0.52$. Our main result is that, during the saturated regime, and independently of the value of $x(0.52 \leqslant x<0.60)$, the ordering process obeys dynamical scaling and the length scale for the domain growth follows an algebraic law $l(t) \sim t^{n}$ with a value of $n$ consistent with the Allen-Cahn exponent, namely, $n=1 / 2$, characteristic of a system with a nonconserved order parameter. In addition we have verified that the movement of a saturated interface proceeds in such a manner such that it covers a constant domain area per unit of time. This feature is of key importance in the Allen-Cahn theory. ${ }^{17,26-28}$ Values of $x$ greater than $x=0.60$ are not studied here since large order-parameter fluctuations associated with a phase transition (at $x=0.64$ for the quench temperature $T=0.25 \mathrm{~J} / K_{B}$ ) take over the system. Complementarily we have investigated the functionality of the prefactor (growth rate) with composition. This has been done indirectly on the length scale obtained from the second moment of the structure factor. The corresponding prefactor $C$ is proportional to the growth rate K. $C$ exhibits a weak linear decrease with an increase in the composition. This has been interpreted in terms of the interplay between clusters of diffusing excess particles, more likely as $x$ increases, and the saturated interfaces which reduces their mobility but keeps unaltered the essential time dependence for the growth law. We recall that this interplay does not proceed via specific interactions. Indeed, the saturated interfaces have to cope with diffusive excess particles during their curvature-driven motion. Nevertheless, the absence of specific interactions reduces the coupling to a simple encounter between interfaces and excess particles which mutually cross their respective trajectories. This does not make the curvature ineffective but may slow down the domain growth.

Experiments in $\mathrm{Cu}_{0.79} \mathrm{Au}_{0.21}$ alloys ${ }^{21}$ reveal that a small amount of excess $\mathrm{Cu}$ atoms has a dramatic effect on the ordering dynamics. One observes a crossover from an algebraic law $\left[l(t) \sim t^{1 / 2}\right]$ for the stoichiometric system $\left(\mathrm{Cu}_{3} \mathrm{Au}\right)$ to a logarithmic growth law. This has been interpreted in terms of the low mobility of the clusters of extra atoms which tend to behave as quenched impurities. ${ }^{21}$ These randomly placed impurities do not generate random fields but modify the local exchange couplings. The corresponding growth behavior has been proposed to be logarithmic. ${ }^{24}$ Later on it was suggested ${ }^{19}$ that the precipitation of impurities at the domain walls may screen direct domain-domain interactions, making the curvature-driven mechanism ineffective. We notice that it is clear from our results that the accumulation of excess $\mathrm{Cu}$ atoms at the interfaces is not directly responsible for the slowing down of the dynamics.

In order to understand the physical origin of the slowing down of the dynamics experimentally observed ${ }^{21}$ and predicted by computer simulations ${ }^{19}$ we extend the discussion to the Ising model with interactions up to NNN's. The Hamiltonian is

$$
\mathscr{H}=J\left[\sum_{\mathrm{NN}} S_{i} S_{j}+\alpha \sum_{\mathrm{NNN}} S_{i} S_{j}\right],
$$

with $J>0$. One can distinguish three different cases: (a) $\alpha$ $=0$ (present work), (b) $0<\alpha<1 / 2$, and (c) $\alpha<0$. We do not consider the case $\alpha>1 / 2$ because it leads to a different ordered structure. In all three cases there is a tendency for the 

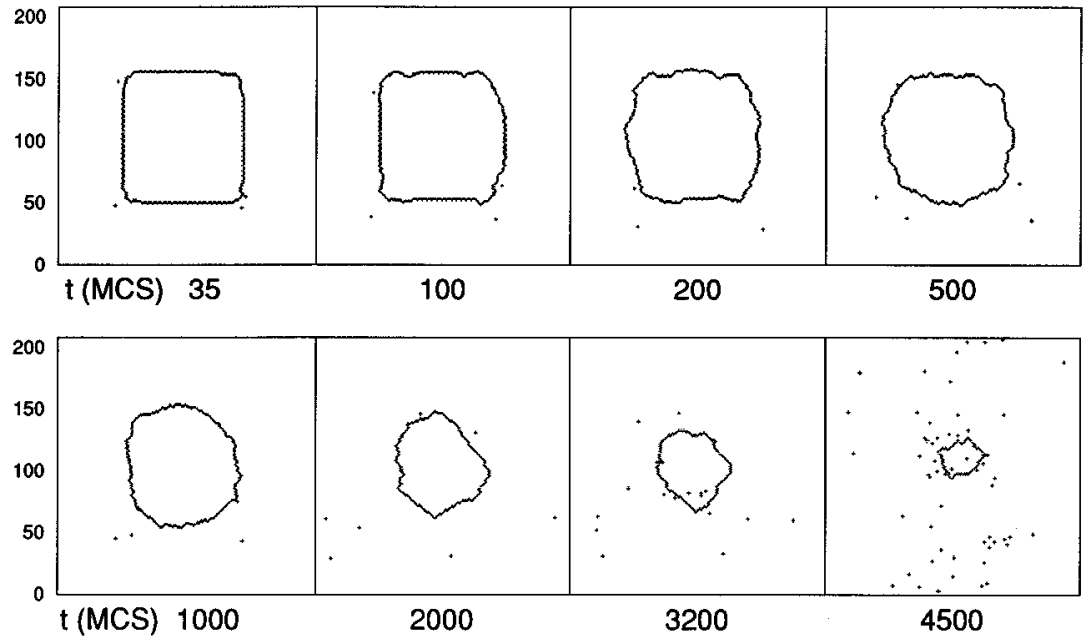

FIG. 8. Evolution of an initially square domain for various instants of time indicated at the bottom of each snapshot. The initial size of the domain is $N=100 \times 100$ and the temperature $T=0.25 J / K_{B}$. The white areas denote ordered particles with energy $-4 J$ whereas the black areas represent disordered particles. excess particles to accumulate at the interfaces but in cases (b) and (c) the dynamics may be substantially different from the present work [case (a)]. The interaction between NNN's introduces a specific interaction between the bulk excess particles themselves and between these excess particles and the saturated interfaces. These interactions, not present in case (a), are repulsive in case (b) and attractive in case (c).

Notice that case (c) is qualitatively similar to the diluted antiferromagnetic Ising model with interactions up to NN's which follows a logarithmic growth ${ }^{19}$ for quenches inside both the antiferromagnetic phase and the region where antiferromagnetic order coexists with a vacancy-rich phase. The $\mathrm{Cu}_{0.79} \mathrm{Au}_{0.21}$ alloy also corresponds to case (c) since $\alpha<0$ is needed to stabilize the $L 1_{2}$ structure. ${ }^{38}$ The similarity between case (c) and the diluted antiferromagnet with interactions up to NN's suggests that the physical origin of the slowing down of the dynamics is the attractive interaction between the saturated interfaces and the excess particles in the bulk.

In Ref. 19 it is shown that for quenches inside the coexistence region, the vacancy-rich phase forms at the antiferromagnetic domain boundaries, giving rise to a screening between the antiferromagnetic domains of opposite order. This makes the curvature-driven pure-system Allen-Cahn growth mechanism ineffective and the growth proceeds via an evaporation-condensation mechanism. We point out that quenches inside the antiferromagnetic phase, where the vacancy-rich phase is not stable, also lead to a logarithmic growth law. ${ }^{19}$ The ingredient present in this last case is the attractive specific interaction between the excess particles in the bulk and the saturated interfaces which we suggest is the physical origin for the dramatic slowing down experimentally found in $\mathrm{Cu}_{0.79} \mathrm{Au}_{0.21}$. On the other hand, the attractive interaction between the excess particles themselves favors the formation of clusters of excess $\mathrm{Cu}$ atoms. As pointed out by Shannon et $a .^{21}$ these clusters have less mobility than a single atom which supports the interpretation of the logarithmic growth behavior of $\mathrm{Cu}_{0.79} \mathrm{Au}_{0.21}$ in terms of an Ising system with quenched random impurities. ${ }^{24,25,39}$ Case (b), which corresponds to a repulsive interaction, will be studied in the future.

Finally we want to emphasize that it is important to dis- tinguish the problem of adsorption of extra particles at the interfaces from that of the interplay between diffusing extra particles inside the bulk and the interfaces when analyzing experimental results. The former has, by itself, no influence on the growth law. The finding of growth laws different from that of the pure Allen-Cahn system is due to the interplay between diffusing particles inside the ordered domains and the interfaces. If this interplay does not involve any specific interaction between free diffusing particles and domain walls, the modifications of the growth law should be accounted for on the prefactor. We suggest that the existence of such interactions is of crucial importance in determining the essential time dependence of the growth law and that it should be taken into consideration when discussing the problem of ordering kinetics in impure systems.

\section{ACKNOWLEDGMENTS}

We are indebted to E. Vives, A. Planes, and C. Frontera for fruitful discussions. We also acknowledge the Fundació Catalana per a la Recerca (FCR) and Centre de Supercomputació de Catalunya (CESCA) for computing facilities and the Comisión Interministerial de Ciencia y Tecnología (CICyT) for financial support (Project No. MAT95-0504). M.P. also acknowledges a fellowship of the Comissionat per a Universitats i Recerca (Generalitat de Catalunya).

\section{APPENDIX A: EVALUATION OF $\boldsymbol{\lambda}_{s}$}

The value of $\lambda_{s}$ can be evaluated as follows. When a particle of the minority component (in our case $B$ ) at the APB with one, two, or three $B-B$ bonds is substituted by an excess $A$ particle from the bulk, the total perimeter length of the APB increases in an amount of 2,0 , or -2 units, respectively. Let $b_{1}, b_{2}$, and $b_{3}$ be the number of $B$ particles at the APB with one, two, and three $B-B$ bonds, respectively, substituted by excess $A$ particles during the adsorption process. The total number of adsorbed particles is then $b_{1}+b_{2}+b_{3}$ and the increase of the total perimeter length is $2 b_{1}-2 b_{3}$. Thus, the density of excess particles at the APB can be written as $\lambda=\left(b_{1}+b_{2}+b_{3}\right) /\left(I^{0}+2 b_{1}-2 b_{3}\right)$ where $I^{0}$ is the total perimeter length of the APB before the adsorption pro- 


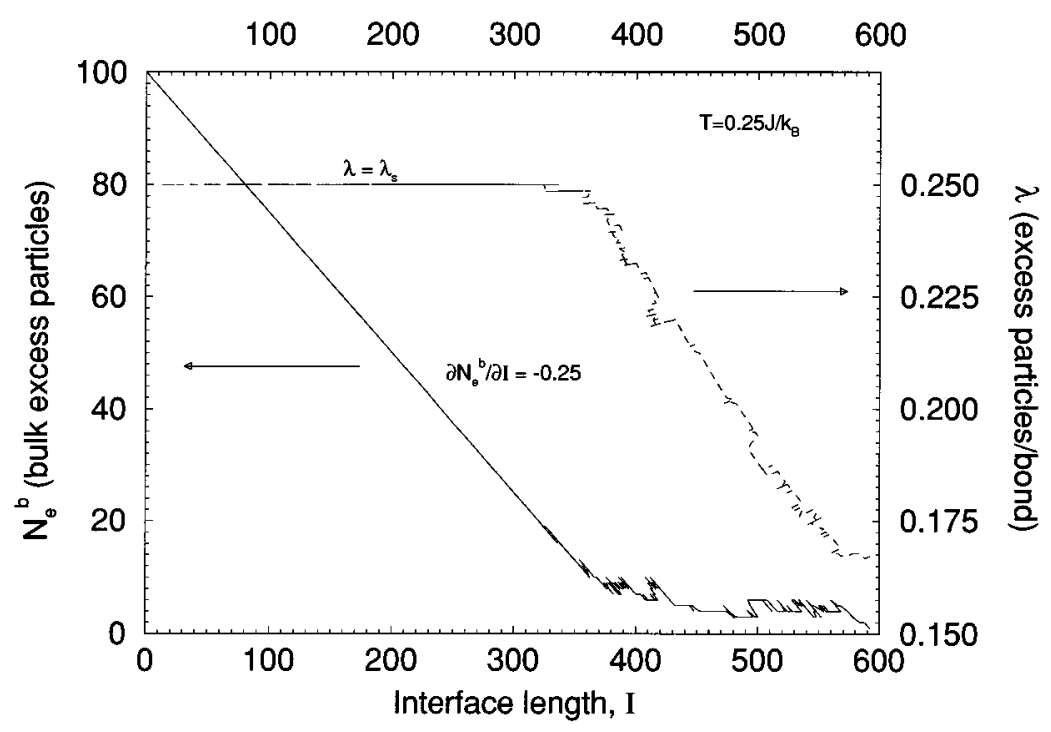

FIG. 9. Number of excess particles in the bulk, $N_{e}^{b}$ (solid line), and density of excess particles at the interface, $\lambda$ (dashed line), both versus the interface length $I$ during the shrinking of the single domain whose evolution in time is shown in Fig. 8. cess. In the absence of interfacial adsorption, the number of $A-A$ bonds at the APB, $N_{A A}^{0}$, is equal to that of $B-B$ bonds, $N_{B B}^{0}=N_{A A}^{0}=\frac{1}{2} I^{0}$. The APB is then saturated when the number of broken $B-B$ bonds, $b_{1}+2 b_{2}+3 b_{3}$, is equal to $N_{B B}^{0}=\frac{1}{2} I^{0}$ and the value of $\lambda_{s}$ is

$$
\lambda_{s}=\frac{b_{1}+b_{2}+b_{3}}{2\left(b_{1}+2 b_{2}+3 b_{3}\right)+2 b_{1}-2 b_{3}}=\frac{1}{4} .
$$

\section{APPENDIX B: CONTROLLED EXPERIMENT- SHRINKAGE OF A SINGLE SQUARE DOMAIN}

In order to have a deeper insight into the evolution of a saturated interface and its intrinsic connection with the ejection of excess particles, we have simulated the time evolution of a single square domain. In the initial configuration all the excess particles are located at the interface. In Fig. 8 we display some snapshots of the evolving domain at selected times. In Fig. 9 we have plotted, versus the total interface length $I$, the density of excess particles at the interface, $\lambda$, and the number of excess particles inside the bulk, $N_{e}^{b}$. In general, it can be observed that, while $\lambda$ is not saturated, the excess particles at the interface are not returned to the bulk and consequently $N_{e}^{b}$ remains constant. Once the interface saturates, the absence of fluctuations allows for a closed re- lationship between $N_{e}^{b}$ and $I$. The reduction of $I$ in 4 units is accompanied by the ejection of an excess particle to the bulk. This is expressed by the slope of the curve $N_{e}^{b}-I$ (Fig. 9) at the saturated regime $\partial N_{e}^{b} / \partial I=1 /(-4)=-0.25$. Nevertheless, it is not impossible that, at the early stages, few excess particles escape through the vertices of the square although the whole interface is not saturated yet. This is because of the extremely high curvature associated with the vertices which favors its local saturation due to a faster shrinking. In spite of this, we may conclude that, at very low temperatures, the probability that an excess particle initially at the interface will be ejected back to the bulk is negligible if the interface is not saturated.

In the case of the domain structure obtained by means of a quench experiment, a broad distribution of curvatures is present during the scaling regime. The interface will first saturate, locally, at the most curved regions of the interface. This early local saturation produces an early ejection of particles reflected by an initial fast increasing of $N_{e}^{b}$ accompanied by the corresponding decreasing of the interface $I$. Nevertheless, such an early ejection is not dominant and the density of excess particles at the interface increases until it raises the saturation. From now on, the system operates in this regime.
${ }^{1}$ J.D. Gunton, M. San Miguel, and P.S. Sahni, in Phase Transitions and Critical Phenomena, edited by C. Domb and J.L. Lebowitz (Academic, New York, 1983), Vol. 8.

${ }^{2}$ H. Furukawa, Adv. Phys. 34, 703 (1985).

${ }^{3}$ O.G. Mouritsen, Int. J. Mod. Phys. B 4, 1925 (1990).

${ }^{4}$ For a recent overview see Dynamics of Ordering Processes in Condensed Matter, edited by S. Komura and H. Furukawa (Plenum, New York, 1988).

${ }^{5}$ K. Binder, Rep. Prog. Phys. 50, 783 (1987).

${ }^{6}$ H. Gilhøj, C. Jeppesen, and O.G. Mouritsen, Phys. Rev. E 52, 1465 (1995).

${ }^{7}$ C. Jeppesen and O.G. Mouritsen, Phys. Rev. B 47, 14724 (1993).
${ }^{8}$ H. Gilhøj, C. Jeppesen, and O.G. Mouritsen, Phys. Rev. Lett. 75, 3305 (1995).

${ }^{9}$ G.C. Wang and T.-M. Lu, Phys. Rev. Lett. 50, 2014 (1983).

${ }^{10}$ S. Katano, M. Iizumi, R.M. Nicklow, and H.R. Child, Phys. Rev. B 38, 2659 (1988).

${ }^{11}$ S.E. Nagler, R.F. Shannon, C.R. Harkless, M.A. Singh, and R.M. Nicklow, Phys. Rev. Lett. 61, 718 (1988).

${ }^{12}$ D.G. Morris, F.M.C. Besag, and R.E. Smallman, Philos. Mag. 29, 43 (1974).

${ }^{13}$ T. Hashimoto, K. Nishihara, and Y. Takeuchi, J. Phys. Soc. Jpn. 45, 1127 (1978). 
${ }^{14}$ Y. Noda, S. Nishihara, and Y.Yamada, J. Phys. Soc. Jpn. 53, 4241 (1984).

${ }^{15}$ S.M. Allen and J.W. Cahn, Acta Metall. 27, 1085 (1979).

${ }^{16}$ M.K. Phani, J.L. Lebowitz, M.H. Kalos, and O. Penrose, Phys. Rev. Lett. 45, 366 (1980).

${ }^{17}$ P.S. Sahni, G. Dee, J.D. Gunton, M. Phani, J.L. Lebowitz, and M. Kalos, Phys. Rev. B 24, 410 (1981).

${ }^{18}$ H.C. Fogedby and O.G. Mouritsen, Phys. Rev. B 37, 5962 (1988), and references therein.

${ }^{19}$ P.J. Shah and O.G. Mouritsen, Phys. Rev. B 41, 7003 (1990).

${ }^{20}$ G.F. Mazenko and O.T. Valls, Phys. Rev. B 33, 1823 (1986).

${ }^{21}$ R.F. Shannon, C.R. Harkless, and S.E. Nagler, Phys. Rev. B 38, 9327 (1988).

${ }^{22}$ K. Oki, H. Sagane, and T. Eguchi, J. Phys. (Paris) Colloq. 38, C7-414 (1977).

${ }^{23}$ T. Ohta, K. Kawasaki, A. Sato, and Y. Enomoto, Phys. Lett. A 126, 93 (1987).

${ }^{24}$ D.A. Huse and C.L. Henley, Phys. Rev. Lett. 54, 2708 (1985).

${ }^{25}$ D.J. Srolovitz and G.N. Hassold, Phys. Rev. B 35, 6902 (1987).

${ }^{26}$ E. Domany and D. Kandel, in Cellular Automata and Modeling of Complex Physical Systems, edited by P. Manneville, N. Boccara, G.Y. Vichniac, and R. Bidaux (Springer-Verlag, Berlin, 1990), p. 98.
${ }^{27}$ P.S. Sahni, G.S. Grest, and S.A. Safran, Phys. Rev. Lett. 50, 60 (1983).

${ }^{28}$ T. Castán and P.-A. Lindgård, Phys. Rev. B 40, 5069 (1989).

${ }^{29}$ K. Huang, Statistical Mechanics, 1st ed. (Wiley, New York, 1963); R.K. Pathria, Statistical Mechanics, 3rd ed. (Pergamon, Oxford, 1984).

${ }^{30}$ K. Binder and D.W. Heermann, Monte Carlo Simulations in Statistical Physics (Springer-Verlag, Berlin, 1988).

${ }^{31}$ Applications of the Monte Carlo Method in Statistical Physics, 2nd ed., edited by K. Binder, Topics in Current Physics, Vol. 36 (Springer-Verlag, Berlin, 1987).

${ }^{32}$ D.W. Heermann, Computer Simulation Methods in Theoretical Physics (Springer-Verlag, Berlin, 1986).

${ }^{33}$ E. Vives and A. Planes, Int. J. Mod. Phys. C 4, 701 (1993).

${ }^{34}$ A. Sadiq and K. Binder, J. Stat. Phys. 35, 517 (1984).

${ }^{35}$ J.W. Cahn and R. Kikuchi, J. Phys. Chem. Solids 20, 94 (1961).

${ }^{36} \mathrm{We}$ have not studied the scaling function as function of $x$. Nevertheless, it is worth mentioning that we used the data obtained for $x=0.50$ to compare both scaling functions, for $x=0.50$ and $x=0.53$, and we did not observe any substantial difference.

${ }^{37}$ J.E. Krzanowski and S.M. Allen, Surf. Sci. 144, 153 (1984); Acta Metall. 34, 1035 (1986).

${ }^{38}$ L.D. Fosdick, Phys. Rev. 116, 565 (1959).

${ }^{39}$ G.S. Grest and D.J. Srolovitz, Phys. Rev. B 32, 3014 (1985). 